\title{
Prototyping Realistic Long-term Human-Robot Interaction for the Study of Agent Migration
}

\author{
K. L. Koay, D. S. Syrdal, W. C. Ho, and K. Dautenhahn, Senior Member, IEEE
}

\begin{abstract}
This paper examines participants' experiences of interacting with a robotic companion (agent) that has the ability to move its "mind" between different robotic embodiments to take advantage of the features and functionalities associated with the different embodiments in a process called agent migration. In particular, we focus on identifying factors that can help the companion retain its identity in different embodiments. This includes examining the clarity of the migration behaviour and how this behaviour may contribute to identity retention. Nine participants took part in a long-term study, and interacted with the robotic companion in the smart house twice-weekly over a period of 5 weeks. We used Narrative-based Integrated Episodic Scenario (NIES) framework for designing long-term interaction scenarios that provided habituation and intervention phases while conveying the impression of continuous long-term interaction. The results show that NEIS allows us to explore complex intervention scenarios and obtain a sense of continuity of context across the long-term study. The results also suggest that as participants become habituated with the companion, they found the realisation of migration signaling clearer, and felt more certain of the identity of the companion in later sessions, and that the most important factor for this was the agent's continuation of tasks across embodiments. This paper is both empirical as well as methodological in nature.
\end{abstract}

\section{INTRODUCTION}

Traditionally robots have had electromechanical embodiments with different appearances ranging from humanlike or animal-like to machine-like [1]. Since their functionalities are often constrained by their embodiments they are often limited to the specific tasks and the particular environments they were designed for. It would be beneficial if the robot's 'mind' (in the sense of memory, decision making, internal states, personality etc.) was able to change its embodiment, when needed, to have access to different capabilities as required by a variety of tasks and working environments [2][3][4][5][6]. This migration would allow the migrating agent to utilise different embodiments, including non-robotic devices such as smart phones, and travel with its user [7], and continue to provide assistance while taking advantage of different embodiments. This would also improve the agent's understanding of, and relationship with the user therefore contributing to a sense of continuity and companionship independently of the agent's specific embodiment [2]. In addition, the agent that inhabits more than one embodiment will be less constrained to a particular physical and information space [5]. Interacting with the user in different embodiments and contexts allows the agent a better understanding of the user, and a stronger sense of contextual

K. L. Koay, D. S. Syrdal and K. Dautenhahn are with the School of Computer Science, University of Hertfordshire, Hertfordshire, Hatfield AL109AB, United Kingdom. (e-mail: (K.L.Koay, D.S.Syrdal, K.Dautenhahn\}@ herts.ac.uk). and situational awareness. Over time the agent would establish its own unique identity, memories and beliefs and should be able to maintain unique and individual relationship with its user, regardless of its embodiments [2][6][8].

From the user's perspective the advantage of migrating an agent is that it would allow continuous interaction with the same agent in different robotic embodiments [9], without having to spend a large amount of time familiarizing and personalizing a variety of different robots or other electronic devices.

Identity retention of a migrating agent in different embodiments is one of the main research challenges we will address in this paper. The fact that an agent can be present in different embodiments may cause confusion for the user to identify the unique identity of their agent [10] in the different embodiments. While specific visual cues, such as a color, or a specific symbol for identifying the individual agent have been proposed [4][11][13][14], this may not be as straightforward with a physically embodied artefact like a robot, in particular if such a robot is what has been termed appearance-constrained [12]. Also, identity retention in terms of a personal agent (i.e. robotic companion), should go beyond simple identification, but should also allow the trust [15] and the relationship [16] that has been built between the user and companion to be retained across embodiments.

We believe it is essential that migrating agents are able to clearly communicate their migration process [4][17][18]. Specific migration signaling can play a key role during a user/-agent habituation period, to convey and establish the concept of the migrating agent to the user, and to distinguish it e.g. from processes where one robot delegates a task to another robot [17]. If the agent is able to help the user establishing the concept of migration, it will be easier for this user to accept and believe in the technology and learn to identify their agent in different embodiments.

Ideally, to address the research issues highlighted above, it is necessary to conduct a long-term human-robot interaction (HRI) study where participants are given the opportunity to use and integrate the migrating agent, as their companion, into their daily routine at their own homes on a long-term basis, beyond habituation and after the novelty effect has worn off. However, it is often not possible to deploy home companion research prototypes in the participants' homes as these systems have not reached the stability and safety levels of commercially available systems and require constant supervision by technical experts to intervene in the event of a

W. C. Ho was with the School of Computer Science, University of Hertfordshire, Hertfordshire, Hatfield AL109AB, United Kingdom. 
system malfunction [19]. Thus, it would be very difficult, if not impossible, to get ethical approval for conducting such a study or consent from the participants to install sensors or make modifications to their homes. Furthermore, deploying such systems at participants' homes would require a significant amount of resources, which are often not feasible.

To overcome these issues, we propose a new scenario prototyping methodology for designing open-ended HRI scenarios that aim to provide participants with immersive long-term interaction experiences similar to those that may have been carried out in their own homes.

Section II shows the specific research questions addressed in this paper. The scenario prototyping methodology will be described in section III together with the scenarios we created and implemented for the user study. The apparatus used in this study is presented in section IV, followed the Method, Results and Conclusion sections in section V, VI and VII respectively.

\section{RESEARCH QUESTIONS}

The goal of this paper is to study issues related to long-term interaction with a migrating agent. In particular, we would like to explore users' perception of the realisation of migration and retention of identity by their companion (i.e. migrating agent) across different embodiments. Realisation of Migration measures the degree to which participants felt that the migration process was successfully communicated by the companion. Retention of Identity measures the degree to which participants felt they were interacting with the same companion in the different embodiments.

These two issues can be formulated into the following research questions:

RQ1. Do participants feel that the process of migration is communicated clearly to the user within the interaction?

RQ2. Do participants feel that they are interacting with the same agent across different embodiments?

RQ3. What are the most important aspects of an agent to communicate identity retention?

To study these issues, we instantiated a set of scenarios in which a human user could interact with one artificial companion which could inhabit one of three different embodiments depending on context.

\section{SCENARIO DEVELOPMENT}

The scenarios used in this study were developed using the Narrative-based Integrated Episodic Scenario (NIES) framework that was intended to provide participants with similar experiences of living and continuous interaction with a robotic migrating companion that is capable of providing physical and cognitive assistance in a real-life domestic environment. The scenarios emphasise long-term and openended interactions to ensure that participants are in charge of their own interactions and that the scenarios are meaningful to their everyday lives. This is to ensure they will gain the necessary experiences to provide well-informed feedback related to the research questions.
This section will briefly introduce the NIES framework followed by a description of the Robot House Scenarios which were developed using NIES framework for this study.

\section{A. Narrative-based Integrated Episodic Scenario Framework}

The Narrative-based Integrated Scenario (NIES) framework was developed for prototyping immersive and open-ended HRI experimental scenarios for long-term studies that are otherwise not feasible due to the constraints associated with the need to embed complex research prototypes into participants' homes.

The NEIS scenario prototyping framework was an improvement over the Integrated Episodic Scenario (IES) framework [20] proposed and validated previously, to provide a means for prototyping immersive episodic interaction scenarios for open-ended HRI study. This is accomplished by using an overall narrative arc to link the episodic scenarios together, forming a chain of meaningful events that participants can relate to and experience during the trials. It provides a more holistic, continuous and targeted interaction experience for the participants with the research prototypes. This is different from the IES framework where the scenarios are isolated, disjoined episodes.

The NIES framework introduces a three-phase structure to guide the design of the main narrative arc, as follows:

1. Demonstrate the research prototypes to the participants.

2. Provide a habituation period for the participants to get used to the experimental environment and daily routine structure while exploring, learning and using the prototypes.

3. Intervene by introducing key events related to the research questions to provide participants with experiences on the issues to elicit well informed feedback based on rich user experiences.

The first two phases are to create a series of scenarios (i.e. a daily routine) for the interaction sessions that would provide participants with experiences similar to how people explore, habituate and utilise new technologies in their own home environment, before introducing narrative-based intervention to elicit their responses.

The third phase focuses on intervention-driven narrative. Specifically, the creation of a series of scenarios that would alter the course of events from the first two phases in order to introduce new situations, which are directly related to the research questions, for the participants to encounter. One of the intervention-driven narratives used in this study is to introduce situations where the companion's embodiment is broken and replaced with a different embodiment.

Interaction scenarios created from the narrative provide real, immersive, open-ended and self-contained interaction sessions that are narratively linking previous and subsequent sessions together allowing for a coherent overall experience with continuity between interaction sessions, an experience similar or close to what they would experience if they had the system installed and used it in their homes. With the interaction scenarios, the participant's goal is to take the role of the main character, acting as themselves, taking part in a 
series of interrelated narrative based interaction sessions where the main character lives and interacts with the technology/system that is being investigated (i.e. a robotic migrating companion) in a naturalistic environment.

\section{B. Overall Scenario}

The overall narrative used in this study was ' $a$ week in the life of a user with their newly acquired robotic companion'. It consisted in total of 9 episodic scenarios created to support the 3 main phases, see TABLE I, to allow the user to explore, habituate and use their new robotic companion in various aspects of possible user activities in open-ended episodic interaction sessions.

Each episodic scenario has its own narrative, but represents particular inter-related key events of the user's life that are related to the robotic companion within the overall narrative time-line to achieve consistency, coherency and realism of the sub-narratives (i.e. close links between different open-ended sessions). These sub-narratives were realized into interaction sessions.

The overall scenario for this study involves a participant, an experimenter whose sole responsibility is taking the role of a technician in the narrative, and the companion (a migrating agent) with its three different robotic embodiments (see Figure 1), which are variations of the "Sunflower" robot. Throughout these scenarios, there is only the companion that the participant interacts with, and it only inhabits one embodiment at any given time. Therefore, if the companion did not inhabit a specific embodiment, the embodiment would be hibernating. While the system could have supported multiple companions sharing these embodiments between them, this was not relevant to this particular usage scenario.

\section{Introduction and Demonstration phase}

This phase represents the first interaction scenario where a user who has just bought a new robotic companion is being introduced to the companion and its embodiments (SF1 Mobile Sunflower and SF2 - Stationary Sunflower) by a technician coming to the user's house.

During this interaction session, the technician demonstrates the companion's functionalities, and shows the participants how to interact with the companion. The participants are taught how to use the companion's Touch Screen Graphical User Interface (GUI) to personalise the companion's (SF1) expressive behaviours, to initiate migration process for the companion to migrate itself between two embodiments (SF1 and SF2), to approve the companion's request to migrate to a different embodiment to achieve its task (e.g. from stationary SF1 to mobile SF2 in order to follow the participant to the kitchen) and to command the companion to carry objects with its tray and to go to various locations in the Robot House. The first session lasted about one hour per participant.

\section{Exploration and Habituation phase}

This phase provided participants with experience of a) a typical daily routine, and b) the fact that the companion uses different embodiments to perform different tasks when assisting the user with his/her activities. Due to the open-ended nature of these interaction sessions, each session lasted
TABLE I. OVERALL SCENARIOS

\begin{tabular}{|l|c|l|}
\hline \multicolumn{1}{|c|}{ Phase } & \multicolumn{2}{|c|}{ Interaction Session } \\
\hline $\begin{array}{l}\text { Introduction and } \\
\text { Demonstration }\end{array}$ & 1 & Introduction and demonstration. \\
\hline \multirow{4}{*}{$\begin{array}{l}\text { Exploration and } \\
\text { Habituation }\end{array}$} & 2 & Breakfast at home \\
\cline { 2 - 3 } & 3 & Late afternoon \\
\cline { 2 - 3 } & 4 & Paperwork afternoon \\
\hline \multirow{4}{*}{ Intervention } & 5 & Departure of mobile embodiment SF1 \\
\cline { 2 - 3 } & 6 & A day with stationary embodiment SF2 \\
\cline { 2 - 3 } & 7 & Arrival of a loan mobile embodiment SF3 \\
\cline { 2 - 3 } & 8 & $\begin{array}{l}\text { Morning routine with, and departure of, } \\
\text { embodiment SF3 }\end{array}$ \\
\cline { 2 - 3 } & 9 & $\begin{array}{l}\text { Return of the original mobile embodiment } \\
\text { SF1 }\end{array}$ \\
\hline
\end{tabular}

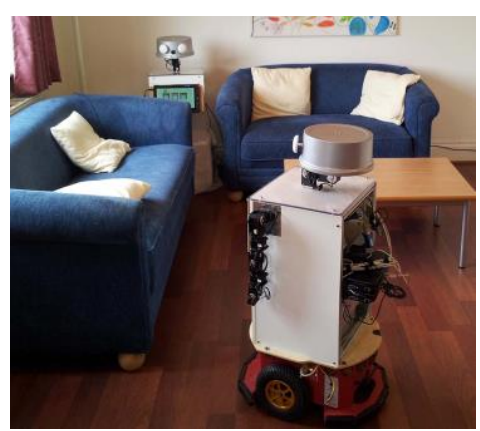

(a)

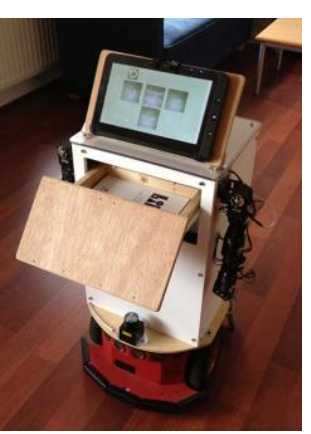

(b)
Figure 1 The three Sunflower robots used in the study. (a) The mobile Sunflower SF1 is located infront of the sofa while the stationary Sunflower SF2 is located behind the sofa, (b) Mobile Sunflower SF3.

between half an hour to an hour depending on participant's behaviours and decisions they made within the trial sessions. The aim was to expose and familiarise the participants with the robotic companion technology focusing on its functionalities and the migration concept through direct exploration and participation.

Breakfast at home session focuses on a morning routine where the narrative begins with the user waking up and then sitting on the sofa. The interaction session begins with the companion approaching and suggesting to the user to have breakfast.

Late afternoon session focuses on a late afternoon routine that begins with the user having just returned home and sitting down on the sofa, resting. The interaction session begins with the companion approaching the user and suggesting to have a drink.

Paperwork in the afternoon session focuses on an after lunch routine that begins with the user returning to the dining table to continue doing paperwork. The interaction session begins with the companion approaching the user and suggesting to have a drink.

\section{Intervention phase}

This phase focuses on exposing participants to scenarios related to research questions in order to elicit informed feedback. A total of five different interaction sessions with 
inter-related narratives were developed, with the goal of enabling participants who have been habituated to the robotic companion in order to experience:

- Losing some of the companion's hardware features and functionalities associated to a particular (simulated) faulty embodiment when it was removed.

- A new physical presence of the companion, associated with the replacement embodiment that has similar physical characteristics to the original one.

- Different robot embodiments (mobile versus static) and similar robot embodiments (mobile versus mobile) performing the same tasks.

- The process of re-acquaintance with the original physical embodiment when it is returned after (simulated) repair.

- The advantages and disadvantages of the migration technology.

The summary for each of the five narratives in chronological ordered are:

Departure of mobile embodiment SF1 session is based on the morning routine participants experienced during the Exploration and Habituation phase. The only difference is the inclusion of an additional intervention narrative that shows the mobile embodiment SF1's LED display panel malfunctioning and the robot being required to be sent back for repair. This involves the technician making a video call to the user and arranging to come and retrieve SF1 for repair. The user then witnesses the technician commanding the companion to migrate from SF1 to the stationary embodiment SF2 before taking SF1 away for repair.

A day with the stationary embodiment SF2 session uses the same morning routine from the Exploration and Habituation phase. The effect of the intervention narrative from the previous session provides a different interaction experience for the participants and their companion in this session. The companion now resides in the stationary embodiment SF2 and has lost all of its physical assistance functionalities due to the lack of mobility. The companion is still able to provide cognitive prosthesis functionalities but instead of exhibiting behaviours associated with the previous embodiment SF1 (approaching the user to show the notification message), now it has to wait for the user to approach embodiment SF2 before it can show the notification message.

The arrival of a loan mobile embodiment SF3 session is based on the late afternoon routine from the Exploration and Habituation phase. The intervention narrative here is to introduce a temporary replacement mobile embodiment SF3 into the overall scenario from the end of this scenario. The majority of the interactions between the user and the companion in this session are still via embodiments SF2, until the very end of the session, when the technician arrives with the replacement embodiment SF3. The user is then asked by the technician to migrate the companion from embodiment $\mathrm{SF} 2$ to SF3 for a test run.
Morning routine with, and departure of, embodiment SF3 session uses the same morning routine. The aim is to provide participants with experiences necessary for them to compare their interaction experiences with the companion when one of the embodiments they are familiar with was replaced with another similar embodiment. In this session, the robotic companion can migrate between stationary embodiment SF2 and the replacement mobile embodiment SF3 and regains its full functionalities including those associated with mobility such as approaching the user to show them notification messages, carry objects for the user from one location to another etc. The first part of the narrative for this session is the same to the morning routine sessions they experienced previously in the Exploration and Habituation phase and the first two sessions in the Intervention phase. This provides participants with a control routine they are familiar with. The familiar routine allows participants to focus on their interaction experiences with the companion after the introduction of the replacement embodiment SF3. The narrative ends with another intervention narrative where the technician making a call informing the user that the embodiment SF1 has been fixed and that he will come to collect embodiment SF3.

Return of the original mobile embodiment SF session was designed to allow participants to re-experience their interaction with SF1 in order for them to compare this experience with their previous interaction experience with SF3. The intervention narrative aims to provide a 'happy ending' to the overall narrative by giving participants a pleasant experience of seeing and interacting with the original mobile embodiment SF1 again. This session uses the morning routine that participants are familiar with plus an added intervention narrative to allow the technician to deliver embodiment SF1 to the user and leave after the user has verified that SF1 is in working order. The morning routine then continues as described previously with the companion utilising both embodiments SF1 and SF2 to assist the user with their activity of daily living. This session creates a comparable interaction experience necessary for the participants to provide constructive feedback with regards to their experiences with the companion when it migrates and utilises embodiments SF1, SF2 and SF3.

\section{APPARATUS}

\section{A. UH Robot House}

The UH Robot House ( $\mathrm{RH})$ is a residential house that has been adapted to provide a real-life domestic setting for the study of HRI [20][21][22][23] and the development of smarthome technology [24] for robot-assisted living [25]. It has been equipped with a real-time electrical energy monitoring system to detect the activation and de-activation of common household electrical appliances such as the TVs, microwaves, computers, table lamps etc. The sensory information is used by the companion to provide appropriate assistance in response to the activity of the user or event as it happens in the environment, i.e. approach the user and offer carrying assistance when they open the fridge door, inform the user when the kettle has boiled or when a visitor rings the doorbell etc. 


\section{B. Sunflower Robot}

The Sunflower robot, shown in Figure 2. was designed and developed as a highly expressive robotic embodiment [22] for the study of robotic home companion in the LIREC project [26]. It has a mobile base, a square body and a cylindrical head. The body of the Sunflower robot is equipped with a diffuse LED display panel in the shoulder as well as a slide-out carrying tray with an integrated GUI on its 'chest'. The robot's head is mounted on a 4-degree of freedom articulated neck and has a non-animated face which was built with two static eyes and a webcam appearing as its nose.

It has four expressive channels and is able to attract the user's attention and provide simple non-verbal feedback, either individually or in combination of using light (multicoloured light signals), sound (midi tunes), and the movements of its head and body. In addition, it can also use its UI to interact with the user. It is integrated into the Robot House computational infrastructure and has the competencies to navigate autonomously, detecting the user's activity (i.e. sitting on the sofa, turning on the kettle, opening the fridge door etc.) and detecting events that are happening in the house (i.e. incoming Skype call, doorbell is ringing), as well as providing cognitive and physical assistance. Its tray can be used to carry objects, an attached tablet computer (GUI) can display messages.

The companion can use any of the Sunflower embodiments shown in Figure 1. The Sunflower robot uses its GUI to communicate with users. For example, in a typical robotinitiated task of reminding the user to have a drink, the robot will approach the user, perform an attention seeking behaviour (i.e. moving its head and body, and blinking its LED panel, while playing short midi tunes) to attract the user's attention, then extend its tray to show the suggestive message on the user interface, with options for the user to agree or reject the suggestion.

Users can also initiate interaction with the companion via the same user interface or by entering their preferences such as drink and food preferences for breakfast etc., as well as personalising the companion's expressive behaviours (lights, colours, movements). The companion can remember the users' settings regardless of which embodiment it has migrated to. The user can ask the companion to go to specific locations (i.e. Kitchen, Living room etc.) in the $\mathrm{RH}$ as well as request it to migrate to a different embodiment via the GUI. Depending on the context or task the companion is going to perform, it may

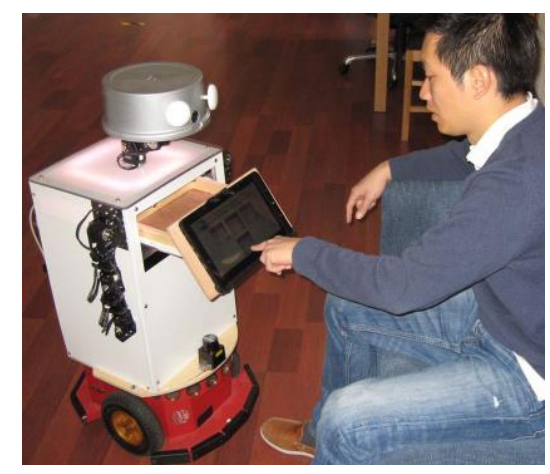

Figure 2. Example of a user interacting with the Sunflower robot. ask the user for permission to migrate to a different embodiment.

The main difference between SF1 and SF2 is that SF2 does not have a mobile base. The main differences between SF1 and SF3 are the lack of an articulated head on SF3 and the GUI that is located on its shoulder. Details of all these differences are shown in TABLE II.

TABLE II. DIFFERENCES BETWEEN THE THREE EMBODIMENTS

\begin{tabular}{|l|l|c|c|c|}
\hline \multirow{4}{*}{$\begin{array}{l}\text { Expressive } \\
\text { Channel }\end{array}$} & $\begin{array}{l}\text { Head } \\
\text { movement }\end{array}$ & Yes & Yes & No \\
\cline { 2 - 5 } & $\begin{array}{l}\text { Body } \\
\text { movement }\end{array}$ & Yes & No & Yes \\
\hline $\begin{array}{l}\text { Navigation } \\
\text { capability }\end{array}$ & Yes & No & Yes \\
\hline \multirow{2}{*}{ GUI } & Location & $\begin{array}{c}\text { Front of } \\
\text { drawer }\end{array}$ & $\begin{array}{c}\text { Front of } \\
\text { drawer }\end{array}$ & $\begin{array}{c}\text { On of } \\
\text { shoulder }\end{array}$ \\
\hline \multirow{2}{*}{ Functionalities } & $\begin{array}{l}\text { Carrying } \\
\text { objects }\end{array}$ & Yes & No & Yes \\
\cline { 2 - 5 } & Navigation & Yes & No & Yes \\
\cline { 2 - 5 } & Skype call & No & Yes & No \\
\hline
\end{tabular}

V. METHOD

\section{A. Measures}

Participant responses to the overall scenario were measured using a Scenario Acceptance Scale which is described in appendix Scenario Evaluation Scale in [20] and intended to measure the participants' acceptance of the robot within the scenario. It consists of 10 Likert scale items, and is scored so that a middle "neutral" response on all items would receive a score of 50 .

Responses to the migration behaviours were measured along two dimensions: Identity Retention, the perception of one agent in the different embodiments; and Migration Realisation, how clear the participants found the behaviour signaling in the process of migration. These questions were used in a previous study and are described in [20]. Participants were asked to complete these after Session 2, 4, 7 and 8.

In addition, participants were asked the following question "Which of the following factors was the one that best communicated it to you that it was the same companion in the different bodies":

- Its knowledge of your interaction history.

- The personalised attention-seeking behaviour.

- The way that it migrated out of, and into its different bodies.

- Its knowledge of your preferences.

- Its ability to continue a task which it had started in a different body

These categories were based on open-ended responses to similar questions in previous studies investigating migration (for instance [20][27][28]) asked after all the 9 sessions had been completed, and was part of an evaluation where the participants were asked to consider the study as a whole. 
Finally, participants were also asked two open-ended questions in the questionnaire: "Did you feel as if you were interacting with the same companion in the different robot bodies?", and "How could it better communicate that it is the same companion?".

\section{B. Procedure}

During the first session, prior to the trial, the experimenter/ facilitator welcomed the participants to the RH introducing himself and a second experimenter whose responsibility is to initiate and monitor the systems during the trial from a small office (i.e. a converted bedroom not used in the study), and also acted as the technician in the narrative. The facilitator then proceeded to introduce and familiarise participants with the RH (i.e. the layout of the house including the location of the dishes, cutlery, cup, tea and coffee etc.), and how to operate the house's electrical appliances.

Below is the procedure for each interaction session.

\section{1) Introduction}

The facilitator would introduce the scenario, grounding the interaction in a narrative which provided context.

An example of a typical short narrative used to set-up the context for a morning interaction session is shown below:

In the introductory session you gave us some preferences for what you would like to do in the early morning. Your robotic companion has these preferences and will apply them when interacting with you.

Now imagine that you have woken up in your bedroom. When you are ready you will come out of the bedroom, sit down on the sofa and log-in to the robot with your user account and password. The robot will then begin today's session.

After giving this information to the participant, the facilitator leaves the participant on the sofa and goes to a facilitator room, to let the participant interact with the robot and the scenario by themselves.

\section{2) Interaction}

This part consisted of the actual interaction scenario. The robot and the participant would interact throughout this scenario without the involvement of the researchers.

For the example above, this part would begin with the robotic companion approaching the participant, seated on the sofa, to suggest breakfast, remind him/her about the hot water in the kettle (if they forgot they were making a hot drink) and notify them about post/parcel/newspaper delivery.

In case the participant needed any assistance from the experimenter during any of the sessions, they were asked to knock 3 times at a designated location on the wall.

\section{3) Post-Interaction}

This phase involved the participant completing the different post-interaction questionnaires and discussing the experiences they just had in the trial with the facilitator. In addition, both researchers would be available to answer any questions that the participant might have about the migrating companion and their interaction. In addition, this phase would also be used to organise future sessions and respond to other issues that might have come up.

\section{Demographics}

There were 9 participants, 6 female and 3 male, recruited via advertisement on the University of Hertfordshire Intranet. The participants were between 21-32 years of age, with a median age of 25 years with an interquartile range of 6 years.

\section{RESULTS}

\section{A. Scenario Acceptance}

The Scenario Acceptance Scores for the Habituation and Manipulation Phase, which focuses on participants' experience of each scenario within the study, is shown in Figure 3. The results suggest that scores on this scale were significantly higher than a 'neutral' score of 50 indicating that the participants found the scenarios believable and provided them with realistic experiences that allowed the participants to suspend their disbelief and immerse themselves into the narratives. There were no significant differences between sessions (Friedman's $\chi^{2}(7)=3.09, p=.87$ ), suggesting that participants responded to the scenario in a consistent manner across sessions.

This suggests that our approach of a Narrative-based Integrated Episodic Scenario framework in a realistic home environment was successful: the environment, the individual narrative within each scenario, the overall narrative between

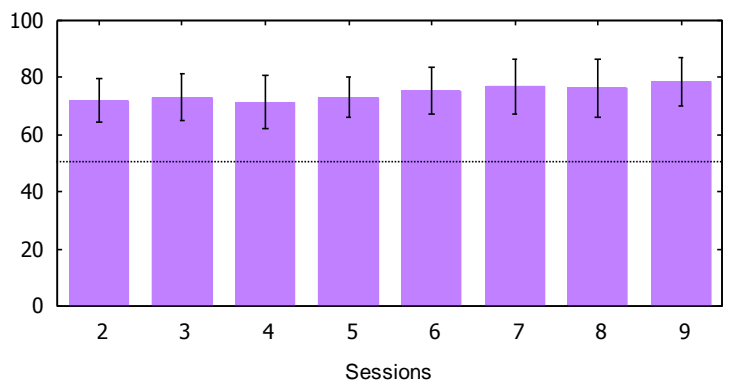

Figure 3. Scenario Acceptance Scores for session from Habituation (2-4) and Manipulation Phase (5-9).

scenarios, the characters and their interactions within the scenarios (i.e. the technician, the robotic companion with its different embodiments and the participants) as a whole provided the realistic interaction experience necessary for the participants to evaluate the system.

\section{B. Migration: Post-Interaction Evaluation}

Participants rated both Migration Realisation and Identity Retention higher as the study progressed (the direction of this trend is described in Figure 4), Friedman tests suggested that this trend was significant for Migration Realisation $\left(\chi^{2}(3)=9.61, p=0.02\right)$, but only approached significance for Identity Retention $\left(\chi^{2}(3)=5.43, \mathrm{p}=0.14\right)$. Post-hoc tests reported in TABLE III and TABLE IV show that the differences were most pronounced between session 8 and the earlier sessions. This trend suggests that not only were participants able to rely more on the cues provided by the system to signal the migration process, but they also felt more certain of the identity of the companion in the later sessions.

\section{Factors for Identity Retention}

Results from the participants' responses to the most important factor that was contributing to the companion's 
identity retention is shown in TABLE V. Interaction context, i.e. the manner in which the companion would begin one task in one embodiment and complete it in another, was rated as most important when contributing to the persistent identity of the agent in different embodiments.

\section{Open-ended Responses}

Responses to the first open-ended question changed slightly over time as well. Overall, participant responses to this item consisted primarily of affirmations that they did feel as if they were interacting with the same companion in the different embodiments. However, 3 participants felt that they had not interacted with the agent enough in the second session, and only one participant referenced this in the fourth session.

Responses to the second open-ended question (how to better communicate identity retention), could be classified roughly into three classes, (1) explicit identification, (2) technical improvements and (3) different model of migration.

Explicit identification was a common theme in Session 2, and suggested that some of the participants wanted the robot to explicitly identify the agent, suggestions for this was a unique identifier for the agent, either in written text, but one participant also suggested a unique face for the agent which could be displayed as an avatar on the touch screen of the robot.

Technical improvements primarily focused on the speed of the migration process, as well as the possibility of foregoing steps in the signaling behaviour in order to have an instantaneous switch from one embodiment to the other when performing tasks.

Finally, some participants rejected the current model of migration and would rather have the agent control all embodiments at the same time. The main motivation for this was ease of use, and the convenience of being able to access specific features of a given embodiment directly from another embodiment.

Some of these results contrast with the results seen from short-term studies such as [5], where explicit identification has been seen as most important. This result is encouraging, especially when using agent migration between robotic embodiments whose appearances are restricted by the functions they are to fulfil, in that coherence of behaviour is a valid way of communicating identity to the user.

\section{CONCLUSION}

By using narrative and persona-driven scenarios, and grounding them in emergent technologies, we were able to create a coherent narrative in which the participants were allowed to interact with an agent migrating between different embodiments. Unlike our previous studies, [20][23], the research presented in this paper shows how one can conduct an 'experimental' manipulation in a narrative study like this. The seamless integration of this manipulation into the narrative made for a more coherent experience of the agent, and allowed us to explore the agent migration in domestic environment to a much greater degree than would have been possible using a constrained or short-term study, despite using technologies that are unstable outside a controlled

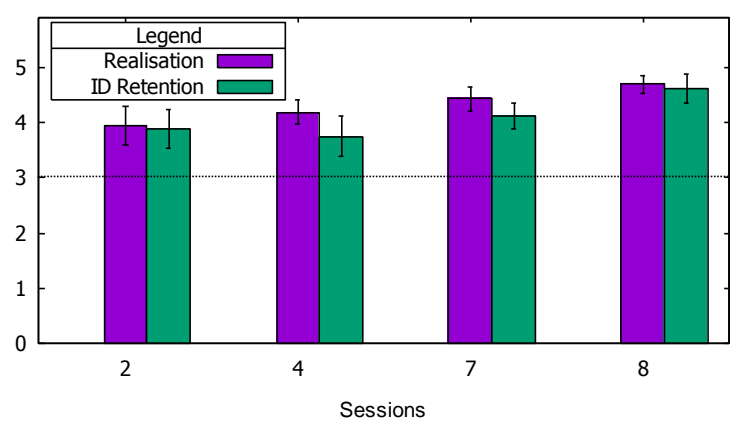

Figure 4. Migration Response Scores for session from Habituation (2-4) and Manipulation Phase (5-9).

TABLE III. WILCOXON LSD POST-HOC TESTS FOR MIGRATION REALISATION

\begin{tabular}{|l|l|c|c|}
\hline & & $\begin{array}{c}\text { Mean } \\
\text { Difference }\end{array}$ & Wilcoxon.p \\
\hline \multirow{3}{*}{ Session 2 } & Session 4 & -0.25 & 0.293 \\
\cline { 2 - 4 } & Session 7 & -0.5 & 0.049 \\
\cline { 2 - 4 } & Session 8 & -0.75 & 0.028 \\
\hline \multirow{2}{*}{ Session 4 } & Session 7 & -0.25 & 0.087 \\
\cline { 2 - 4 } & Session 8 & -0.25 & 0.049 \\
\hline Session 7 & Session 8 & 0 & 0.231 \\
\hline
\end{tabular}

TABLE IV. WILCOXON LSD POST-HOC TESTS FOR IDENTITY RETENTION

\begin{tabular}{|l|l|c|c|}
\hline & & $\begin{array}{c}\text { Mean } \\
\text { Difference }\end{array}$ & Wilcoxon.p \\
\hline \multirow{3}{*}{ Session 2} & Session 4 & 0.125 & 0.500 \\
\cline { 2 - 4 } & Session 7 & -0.25 & 0.425 \\
\cline { 2 - 4 } & Session 8 & -0.75 & 0.121 \\
\hline \multirow{2}{*}{ Session 4 } & Session 7 & -0.375 & 0.293 \\
\cline { 2 - 4 } & Session 8 & -0.375 & 0.101 \\
\hline Session 7 & Session 8 & -0.5 & 0.102 \\
\hline
\end{tabular}

TABLE V. MOST IMPORTANT FACTOR FOR IDENTITY RETENTION

\begin{tabular}{|l|c|}
\hline Factor & Participants \\
\hline Accessing Interaction History & 2 \\
\hline Personalised Attention Seeking Behaviour & 1 \\
\hline Migration Realisation & 0 \\
\hline Personalised Preferences & 0 \\
\hline Sense of Context/Task Continuation & 5 \\
\hline
\end{tabular}

development environment.

With the NIES framework we were able to explore complex intervention scenarios and obtain results for migrating agents suggesting that the sense of context/task continuation factors can be used to help a migrating agent to retain its identity in different robot embodiments that may not have properties that are similar or replicable. Signaling the migration process (realisation of migration) may not be the most important factor for our participants at the end of the study when compared to other factors shown in TABLE V. This may be due to the fact that the migration process can be easily missed if one is not paying attention, a typical situation in a domestic environment where people are easily distracted. 
Hence it is not as reliable when compared to other rated factors such as the recorded interaction history between participants and the companion, the personalised attention seeking behaviour, and the sense of context/task continuation for identifying the identity of the agent. However, the results did indicate that participants found that migration signaling became clearer and more meaningful as they habituated with the companion and the concept of agent migration [17].

The main limitation to our approach is the small sample size caused by the large amount of resources required to maintain both the prototype technologies as well as to structure the narrative. However, the need for participants to have at least two one hour interactions sessions per week limited the number of participants we can practically accommodate in the Robot House during a working week. This may limit the generalisation of results, but even so, the findings suggest that studies intended to explore and prototype domestic technologies like robot companions, can use complex, meaningful and engagement narratives, even at this early stage of technology development, using the Narrative-based Integrated Episodic Scenario (NIES) framework.

\section{ACKNOWLEDGMENT}

The work described in this paper was conducted within the EU Integrated Projects, LIREC (Living with Robots and Interactive Companions). Funded by the European Commission under FP7-ICT under contract FP7-215554.

\section{REFERENCES}

[1] M. L. Walters, D.S. Syrdal, K. Dautenhahn, R. Te Boekhorst, and K. L. Koay, "Avoiding the uncanny valley: robot appearance, personality and consistency of behavior in an attention-seeking home scenario for a robot companion," Autonomous Robots, vol.24, no.2, 159-178, 2008.

[2] K. Ogawa, and T. Ono, "Itaco: Constructing an emotional relationship between human and robot," in Proc. of RO-MAN 2008, pp.35-40.

[3] M. Imai, T. Ono and T. Etani, "Agent migration: communications between a human and robot," in Proc. IEEE International Conference on Systems, Man, and Cybernetics, vol. 4, pp. 1044-1048, Tokyo, 1999.

[4] K. L. Koay, D. S. Syrdal, K. Dautenhahn, K. Arent, L. Malek, and B. Kreczmer, "Companion Migration - Initial Participants' Feedback from a Video-Based Prototyping Study", Mixed Reality and Human-Robot Interaction, Springer, Series "Intelligent Systems, Control and Automation: Science and Engineering", vol. 47, Xiangyu Wang (Ed.), pp133-151, 2011.

[5] B. R. Duffy, G. M. O'Hare, A. N. Martin, J. F. Bradley, and B. Schon, "Agent chameleons: Agent minds and bodies," in Proc. CASA 2003, pp.118-125.

[6] K. Hassani and W.S. Lee, "On designing migrating agents: from autonomous virtual agents to intelligent robotic systems," in SIGGRAPH Asia 2014 Autonomous Virtual Humans and Social Robot for Telepresence (SA '14), ACM, Article 7, 10 pages, 2014.

[7] R. Aylett, M. Kriegel, I. Wallace, E. Márquez Segura, J. Mecurio, S. Nylander and P. Vargas, "Do I remember you? Memory and identity in multiple embodiments," in Proc. RO-MAN 2013, pp. 143-148.

[8] K. F. Macdorman and S. J. Cowley, "Long-term Relationships as a Benchmark for Robot Personhood," in Proc. RO-MAN 2006, pp.378-383.

[9] P. F. Gomes, E. M. Segura, H. Cramer, T. Paiva, A. Paiva, and L. E. Holmquist, "ViPleo and PhyPleo: artificial pet with two embodiments," in Proc. ACE 2011, ACM, pp.1-8.

[10] B. Tomlinson, M. L. Yau, and E. Baumer, "Embodied mobile agents," in Proc. AAMAS , pp.969-976, 2006.
[11] G. M. P. O’Hare, B. R. Duffy, B. Schön, A. N. Martin, and J. F. Bradley, "Agent chameleons: Virtual agents real intelligence," In Intelligent virtual agents, pp.218-225, 2003.

[12] C. L. Bethel, and R. R. Murphy, "Survey of non-facial/non-verbal affective expressions for appearance-constrained robots," in IEEE Transactions on Systems, Man, and Cybernetics, Part C: Applications and Reviews, vol. 38, no.1, 83-92, 2008.

[13] A. Martin, B. Duffy, G. O’Hare, B. Schön, and J. Bradley, "Empowering Agents within Virtual Environments," in Proc. of the IEEE Systems, Man, and Cybernetics UKRI 3rd Workshop on Intelligent Cybernetic Systems, IEEE Computer Society, Los Alamitos, 2004.

[14] S.Yasuyuki, and M. Kenji, “AgentSalon: facilitating face-to-face knowledge exchange through conversations among personal agents," in Proc. of the Fifth International Conference on Autonomous Agents, pp.393-400, 2001.

[15] M. Salem, G. Lakatos, F. Amirabdollahian, K. Dautenhahn, "Would You Trust a (Faulty) Robot? Effects of Error, Task Type and Personality on Human-Robot Cooperation and Trust," in Proc. HRI 2015, pp. 141-148.

[16] N. Martelaro, M. Shiloh, W. Ju, "Tell Me More: Designing HRI to encourage more trust, disclosure and companionship," in Proc. HRI 2016, pp. 181-188.

[17] K. L. Koay, D. S. Syrdal, M. L. Walters, and K. Dautenhahn, "A User Study on Visualization of Agent Migration between Two Companion Robots," in Proc. HCII 2009, pp. 219-226.

[18] K. Arent and B. Kreczmer, "Identity of a companion, migrating between robots without common communication modalities: Initial results of VHRI study," in Proc. MMAR 2013, pp. 109-114.

[19] J. Gallego-Pérez, M. Lohse, and V. Evers, "Robots to motivate elderly people: present and future challenges," in Proc. RO-MAN 2013, pp.685-690.

[20] D. S. Syrdal, K. Dautenhahn, K. L. Koay, and W. C. Ho, "Views from within a narrative: Evaluating long-term human-robot interaction in a naturalistic environment using open-ended scenarios," Cognitive computation, vol.6, no.4, pp.741-759, 2014.

[21] K. L. Koay, D. S. Syrdal, M. Ashgari-Oskoei, M. L. Walters, and K. Dautenhahn, "Social Roles and Baseline Proxemic Preferences for a Domestic Service Robot," Int. J. of Social Robotics vol. 6, pp.469-488, 2014.

[22] K. L. Koay, G. Lakatos, D. S. Syrdal, M. Gácsi, B. Bereczky, K. Dautenhahn, A. Miklósi, and M. L. Walters, "Hey! there is someone at your door. A hearing robot using visual communication signals of hearing dogs to communicate intent," in Proc. of the IEEE Symposium on Artificial Life, pp.90-97, 2013.

[23] D. S. Syrdal, K. Dautenhahn, K. L. Koay, and W. C. Ho, "Integrating constrained experiments in long-term human-robot interactions using task-and scenario-based prototyping," The Information Society Special Issue: Beyond Industrial Robotics: Social Robots Entering Public and Domestic Spheres, vol. 31, no. 3, pp.265-283, 2015.

[24] I. Duque, K. Dautenhahn, K. L. Koay, I. Willcock, and B. Christianson, "Knowledge-driven user activity recognition for a smart house. Development and validation of a generic and low-cost, resourceefficient system," in Proc. the international conference on Advances in Computer-Human Interactions, pp. 141-146, 2013.

[25] J. Saunders, D. Syrdal, K. L. Koay, N. Burke and K. Dautenhahn, "Teach Me - Show Me'- End-user personalisation of a smart home and companion robot," in IEEE Transactions on Human-Machine Systems, vol. 46, no. 1, pp.27-40, 2015.

[26] LIREC - Living with Robots and Interactive Companions. Available: http://www.lirec.eu/. [Accessed: 2- March- 2016].

[27] D. S. Syrdal, K. L. Koay, M. L. Walters, and K. Dautenhahn, "The boyrobot should bark! -children's impressions of agent migration into diverse embodiments," in Proc. of New Frontiers of Human-Robot Interaction, a symposium at AISB, 2009.

[28] K. L. Koay, D. S. Syrdal, M. L. Walters, and K. Dautenhahn, “A user study on visualization of agent migration between two companion robots," in Proc. of Human-Computer Interaction International (HCII 2009). 